\title{
Application of Adaptive Kalman Filter in Online Monitoring of Mine Wind Speed
}

\author{
De Huang ${ }^{1,2, *}$, Jian Liu ${ }^{1,2, *}$, Lijun Deng ${ }^{1,2}$, Xuebing $\mathrm{Li}^{2,3}$ and Ying Song ${ }^{2,4}$ \\ 1 College of Safety Science \& Engineering, Liaoning Technical University, Huludao 125105, China; \\ anheihb03dlj@163.com(L.D.) \\ 2 Key Laboratory of Mine Thermo-motive Disaster and Prevention, Ministry of Education, Huludao 125105, \\ China \\ 3 School of Safety Engineering, North China Institute of Science and Technology, Beijing 101601, China; \\ 1xb19871201@126.com(X.L.) \\ 4 College of Management Science and Engineering, Shandong Technology and Business University, Yantai \\ 264005, China; songying927@163.com(Y.S.) \\ * Correspondence: hunanhd@163.com (D H); liujian@lntu.edu.cn (J.L.); Tel.: +86-186-9899-4238 (D.H.)
}

\begin{abstract}
The underground complicated testing environment and the fan operation instability cause large random errors and outliers of the wind speed signals. The outliers and large random errors result in distortion of mine wind speed monitoring, which possesses safety hazards in mine ventilation system. Application of Kalman filter in velocity monitoring can improve the accuracy of velocity measurement and eliminate the outliers. Adaptive Kalman Filter was built by automatically adjusting process noise covariance and measurement noise covariance depending on the differences between measured and expected speed signals. We analyzed the fluctuation of airflow flow using data of wind speed flow and distribution characteristics of the tunnel obtained by the Laser Doppler Velocimetry system (LDV) studies. A state-space model was built based on the tunnel airflow fluctuations and wind speed signal distribution. The adaptive Kalman Filter was calculated according to the actual measurement data and the Expectation Maximization (EM) algorithm. The adaptive Kalman filter was used to shield fluid pulsation while preserving system-induced fluctuations. Using the Kalman filter to treat offline wind speed signal acquired by LDV, the reliability of Kalman filter wind speed state model and the characteristics of adaptive Kalman Filter were investigated. Results showed that the adaptive Kalman filter effectively eliminated the outliers and reduced the root-mean-squares error (RMSE), and the adaptive Kalman filter had better performance than the traditional Kalman filter in eliminating outliers and reducing RMSE. Field experiments in online wind speed monitoring were conducted using the optimized adaptive Kalman Filter. Results showed that adaptive Kalman filter treatment could monitor the wind speed with smaller RMSE compared with LVD monitor. The study data demonstrated that the adaptive Kalman filter is reliable and suitable for online signal processing of mine wind speed monitor.
\end{abstract}

Keywords: mine wind speed; Laser doppler velocimetry; Kalman filter; expectation maximization algorithm; online monitoring.

\section{Introduction}

One of the basic conditions for mine safety production is a reliable mine ventilation system, especially in coal mine production. Accurate ventilation parameters must be tested to ensure effective ventilation. The air volume is one of the most important parameters for mine ventilation [1]. At present, mine air volume is calculated by testing the average wind speed at the tunnel test point section and the test point cross-sectional area. Therefore, the mine air volume is actually the average wind speed of the roadway test point section, or the average wind speed is obtained by measuring the average dynamic pressure value of the tunnel section through the piezometer. In order to obtain an accurate average wind speed in the tunnel section, some scholars conducted related testing and 
simulation study on the average value of single-point test of tunnel by using laser Doppler velocimeter and CFD simulation theory [2-4]. The results showed that there was a positive correlation relationship between the wind speed at certain points in the roadway section and the average wind speed in the roadway section under certain conditions. The above study analyzed the relationship between turbulent pulsation characteristics and cross-section wind speed and average wind speed. However, the accuracy of the average wind speed is determined by the precision and accuracy of the single point test data.

Therefore, it is necessary to get precise and accurate wind speed for accurate estimation of section air volume in the mine. Sensors are usually used for mine data collection. The results obtained by wind speed sensor monitoring often have large random errors and fluctuations, and outliers [5]. Filtering is the common method for sensor signal processing [6, 7]. There is random white noise in the mine wind speed monitoring, which is consistent with the use conditions of the Kalman filter [8, 9]. Kalman filters are used widely in various fields [10-13]. Kalman filter can treat the disordered and fluctuating data collected by the roadway wind speed sensors to get valid mine wind speed data for analysis and therefore improve the accuracy of wind speed monitor by effectively shielding invalid measurement values.

We analyzed the fluctuation of airflow flow using data of wind speed flow and distribution characteristics of the tunnel obtained by the LDV studies. A state-space model was built based on the tunnel airflow fluctuations and wind speed signal distribution. The adaptive Kalman Filter was calculated according to the actual measurement data and the EM algorithm. Using the Kalman filter to treat offline wind speed signal acquired by LDV, the reliability of Kalman filter wind speed state model and the characteristics of adaptive Kalman Filter were investigated. Field experiments in online wind speed monitoring were conducted using the optimized adaptive Kalman Filter, the applicability of the adaptive Kalman filter in online monitoring of mine wind speed were verified.

\section{Adaptive Kalman filter}

\subsection{State space model}

The flow of wind in the underground tunnel is very complicated. Random changes in the state of the structure and unknown factors such as the operation of the mine cars lead to unstable airflow conditions, which would make large wind speed random errors and random fluctuations at the location of the wind speed monitoring sensors. It is difficult to identify whether alterations are turbulent pulsations or disturbances caused by system movement. The state space model is important for improving the performance of the Kalman filter. Kalman filter can filter the monitored data by shielding the fluctuations caused by turbulent pulsations so that the output of each monitoring moment is close to the true situations. Assuming that $\mathrm{T}$ is sampling interval, the Kalman filter model assumes the true state at time $t$ is evolved from the state at $(t-1)$ according to [14]:

$$
\boldsymbol{x}_{t}=\boldsymbol{A}_{t} \boldsymbol{x}_{t-1}+\boldsymbol{B}_{t} u_{t-1}+\boldsymbol{\omega}_{t-1}
$$

where $\boldsymbol{A}_{t}$ is a state transition model from the state of the previous moment $\boldsymbol{x}_{t-1}$ to the state of the moment $\boldsymbol{x}_{t} ; \boldsymbol{B}_{t}$ is the control-input model, which is applied to the control vector $u_{t} ; \boldsymbol{\omega}_{t}$ is the process noise which is assumed that the mean normal value of zero and the multivariate normal distribution of the covariance $\boldsymbol{Q}_{t}: \boldsymbol{\omega}_{t} \sim \boldsymbol{N}\left(0, \boldsymbol{Q}_{t}\right)$;

At time $t$ a measurement $\boldsymbol{z}_{t}$ of the true state $\boldsymbol{x}_{t}$ is made according to

$$
\boldsymbol{z}_{t}=\boldsymbol{H}_{t} \boldsymbol{x}_{t}+\boldsymbol{v}_{t}
$$

where $\boldsymbol{H}_{t}$ is the observation model which maps the true state space into the observed space; $\boldsymbol{v}_{t}$ is the observation noise which is assumed to be zero mean Gaussian white noise with covariance $\boldsymbol{R}_{t}: \boldsymbol{v}_{t} \sim \boldsymbol{N}\left(0, \boldsymbol{R}_{t}\right)$. 
The previous time estimate will be denoted as $\hat{\boldsymbol{x}}_{t}^{-}$, where the "hat" denotes estimate, and the "super minus" is a reminder that this is the best estimate prior to assimilating the measurement at time of $t$. The Kalman filtering base equations are given by:

$$
\left\{\begin{array}{l}
\hat{\boldsymbol{x}}_{t}^{-}=\boldsymbol{A}_{t} \hat{\boldsymbol{x}}_{t-1}+\boldsymbol{B}_{t} u_{t-1} \\
\hat{\boldsymbol{p}}_{t}^{-}=\boldsymbol{A}_{t} \boldsymbol{p}_{t-1} \boldsymbol{A}_{t}^{T}+\boldsymbol{Q}_{t} \\
\boldsymbol{K}_{t}=\hat{\boldsymbol{p}}_{t}^{-} \boldsymbol{H}_{t}^{T}\left(\boldsymbol{H}_{t} \boldsymbol{P}_{t}^{-} \boldsymbol{H}_{t}^{T}+\boldsymbol{R}_{t}\right)^{-1} \\
\hat{\boldsymbol{x}}_{t}=\hat{\boldsymbol{x}}_{t}^{-}+\boldsymbol{K}_{t}\left(\boldsymbol{z}_{t}-\boldsymbol{H}_{t} \hat{\boldsymbol{x}}_{t}^{-}\right) \\
\boldsymbol{p}_{t}=\left(\boldsymbol{I}-\boldsymbol{K}_{t} \boldsymbol{H}_{t}\right) \boldsymbol{p}_{t}^{-}
\end{array}\right.
$$

where $\hat{\boldsymbol{p}}_{t}^{-}$is the priori estimate error covariance; $\boldsymbol{K}_{t}$ is the Kalman gain.

In this state space model, $\boldsymbol{A}_{t}=1, \boldsymbol{B}_{t}=0, \boldsymbol{H}_{t}=1, \boldsymbol{Q}_{t}=\boldsymbol{Q}, \boldsymbol{R}_{t}=\boldsymbol{R}$, where $\boldsymbol{Q}$ and $\boldsymbol{R}$ are constants. And the base equations are changed to:

$$
\left\{\begin{array}{l}
\hat{\boldsymbol{p}}_{t}^{-}=\boldsymbol{p}_{t-1}+\boldsymbol{Q} \\
\boldsymbol{K}_{t}=\hat{\boldsymbol{p}}_{t}^{-}\left(\boldsymbol{P}_{t}^{-}+\boldsymbol{R}\right)^{-1} \\
\hat{\boldsymbol{x}}_{t}=\hat{\boldsymbol{x}}_{t-1}+\boldsymbol{K}_{t}\left(z_{t}-\hat{\boldsymbol{x}}_{t-1}\right) \\
\boldsymbol{p}_{t}=\left(\boldsymbol{I}-\boldsymbol{K}_{t}\right) \boldsymbol{p}_{t}^{-}
\end{array}\right.
$$

where $\boldsymbol{z}_{t}=\boldsymbol{v}_{t}$ is the measured instantaneous wind speed at the test point at time $t$, the unit is $\mathrm{m} / \mathrm{s}$. $\hat{\boldsymbol{x}}_{t}=\hat{\boldsymbol{v}}_{t}$ is the test point to estimate the instantaneous wind speed at time $t$, that is, the instantaneous point wind speed after Kalman filter processing, the unit is m/s.

\subsection{Adaptive parameter adjustment}

Process noise covariance $\boldsymbol{Q}$ and measurement noise covariance $\boldsymbol{R}$ are important for Kalman filter performance improvement [8]. Inappropriate $\boldsymbol{R}$ and $\boldsymbol{Q}$ make poor performance of the Kalman filter in eliminating outliers and reducing random errors [15]. In practical applications, the $\boldsymbol{R}$ and $\boldsymbol{Q}$ are difficult to be determined. Only by constantly adjusting $\boldsymbol{R}$ and $\boldsymbol{Q}$ can the Kalman filter achieve the best performance.

EM algorithm is a method for finding maximum likelihood estimation parameters from incomplete data sets [16]. This method is widely used to deal with incomplete data such as defects, truncation, and noise [17]. According to the deviation between the observed and estimated values, Kalman's orthogonality and wind speed estimation error, using the EM algorithm to achieve process noise covariance and measurement noise covariance adaptive adjustment can improve Kalman's filtering performance.

The purpose of the Kalman-EM algorithm is to find a set of parameters $\theta=\left(\boldsymbol{Q}, \boldsymbol{R}, \mu_{0}, \Sigma_{0}\right)$ that maximize the probability $P\left(z_{0: T-1} ; \theta\right)$ of Kalman performance. Where $\mu_{0}, \Sigma_{0}$ are the initial mean and variance, respectively.

Using $\mu_{t \mid 0: t-1}$ to represent $\boldsymbol{E}\left(x_{t} \mid \boldsymbol{z}_{0: T-1}\right)$, and $\sum_{t \mid 0: t}$ to represent $\operatorname{Var}\left(\boldsymbol{x}_{t} \mid \boldsymbol{z}_{0: T-1}\right)$, where $\boldsymbol{E}(\zeta)$ is the mathematical expectation of $\zeta$ and $\operatorname{Var}(\zeta)$ is the variance of $\zeta$. Then the Kalman filter recursively forwards to obtain: 


$$
\left\{\begin{array}{l}
\mu_{t \mid 0: t-1}=\mu_{t-1 \mid 0: t-1} \\
\sum_{t \mid 0: t-1}=\sum_{t-1 \mid 0: t-1}+\boldsymbol{Q} \\
\boldsymbol{K}_{t}=\sum_{t \mid 0: t-1}\left(\sum_{t \mid 0: t-1}+\boldsymbol{R}\right)^{-1} \\
\mu_{t \mid 0: t}=\mu_{t \mid 0: t-1}+\boldsymbol{K}_{t}\left(z_{t}-\mu_{t \mid 0: t-1}\right) \\
\sum_{t \mid 0: t}=\left(\boldsymbol{I}-\boldsymbol{K}_{t}\right) \sum_{t \mid 0: t-1}
\end{array}\right.
$$

where $\mu_{1 \mid 0}=\mu_{0}, \quad \sum_{1 \mid 0}=\sum_{0}$. To get $\max _{\theta} P\left(z_{0: T-1} ; \theta\right)$, need to perform backward recursion:

$$
\left\{\begin{array}{l}
\boldsymbol{J}_{t-1}=\sum_{t-1 \mid 0: t-1} \sum_{t \mid 0: t-1}{ }^{-1} \\
\mu_{t-1 \mid 0: T-1}=\mu_{t-1 \mid 0: t-1}+\boldsymbol{J}_{t-1}\left(\boldsymbol{x}_{t \mid 0: T-1}-\boldsymbol{x}_{t \mid 0: t-1}\right) \\
\sum_{t-1 \mid 0: T-1}=\sum_{t-1 \mid 0: t-1}+\boldsymbol{J}_{t-1}\left(\sum_{t \mid 0: T-1}-\sum_{t \mid 0: t-1}\right) \boldsymbol{J}_{t-1}{ }^{\mathrm{T}}
\end{array}\right.
$$

This gives the expressions of $\boldsymbol{Q}$ and $\boldsymbol{R}$ :

$$
\begin{gathered}
\boldsymbol{Q}=\frac{1}{T-1} \sum_{t=0}^{T-2}\left(\mu_{t+1 \mid 0: T-1}-\mu_{t \mid 0: T-1}\right)\left(\mu_{t+1 \mid 0: T-1}-\mu_{t \mid 0: T-1}\right)^{\mathrm{T}}+\sum_{t \mid 0: T-1}+\sum_{t+1 \mid 0: T-1}-\sum_{t+1 \mid 0: T-1} \boldsymbol{J}_{t}^{\mathrm{T}}-\boldsymbol{J}_{t} \sum_{t+1 \mid 0: T-1} \\
\boldsymbol{R}=\frac{1}{T} \sum_{t=0}^{T-1}\left(z_{t}-\mu_{t \mid 0: T-1}\right)\left(z_{t}-\mu_{t \mid 0: T-1}\right)^{\mathrm{T}}+\sum_{t \mid 0: T-1}
\end{gathered}
$$

The instantaneous wind speed can be obtained by recursively calculating the basic equation (4) of the Kalman filter using the parameters calculated by equations (7) and (8).

$$
\left\{\begin{array}{l}
\left|E\left(z_{t}-\hat{\boldsymbol{x}}_{t-1}\right)\right|<0.05 \\
\left|\boldsymbol{R}+P_{t}^{-}-\operatorname{Cov}\left(z_{t}-\hat{\boldsymbol{x}}_{t-1}\right)\right|<0.005
\end{array}\right.
$$

If the constraint condition of (9) is satisfied, the test value $\boldsymbol{z}_{t}$ at time $t$ is a normal value, otherwise $z_{t}$ is outlier, at this time, the estimated value is used as the true value at time $t$ to update the process noise covariance and the measurement noise covariance.

\section{Experiment}

The mine wind speed monitoring is interfered by the complex and variable test environment, there are many unknown disturbance factors in the wind speed monitoring signal in actual production. The wind speed signal that monitored is difficult to determine the performance of the Kalman filter. Therefore, the experimental monitoring of the wind speed signal is carried out using a laser Doppler velocimetry system with high measurement accuracy and less influence from external disturbance factors. The obtained offline signals were processed by a conventional Kalman filter and an adaptive Kalman filter for comparing the performance of the Kalman filter in reducing random errors and rejecting outliers. But in the production process, off-line wind speed signal processing is difficult to meet the needs of real-time monitoring of mine wind speed, real-time filtering of wind speed monitor signals is required. Therefore, the experimental mine was used for the field test to determine the feasibility and application performance of the adaptive Kalman filter in mine wind speed online monitoring.

\subsection{LDV experimental model}

The mine wind speed LDV test experimental equipment and model are shown in Figure 1. A square experimental model was used. The model had a proportion of 1:20, a section inner diameter of $0.2 \mathrm{~m}$ and a length of $15 \mathrm{~m}$. To trace the airflow, the smoke with an average particle diameter of fewer than $2 \mu \mathrm{m}$ is used as the tracer particles. Speed measurement precision reaches $0.1 \%$. During 
the experiment, the laser transmitter emitted 6 beams of laser and the laser was combined into 3 beams of laser through a probe, the colors were green, blue and purple, respectively, for testing fluid velocity in the $\mathrm{X}, \mathrm{Y}$, and $\mathrm{Z}$ directions, where $\mathrm{X}$ is the flow direction, $\mathrm{Y}$ is the extension direction, and $\mathrm{Z}$ is the vertical direction. The laser probes were fixed on the $3 \mathrm{D}$ coordinate frame and the pitch of the probe was controlled by a computer, and movement accuracy was up to $0.001 \mathrm{~mm}$.

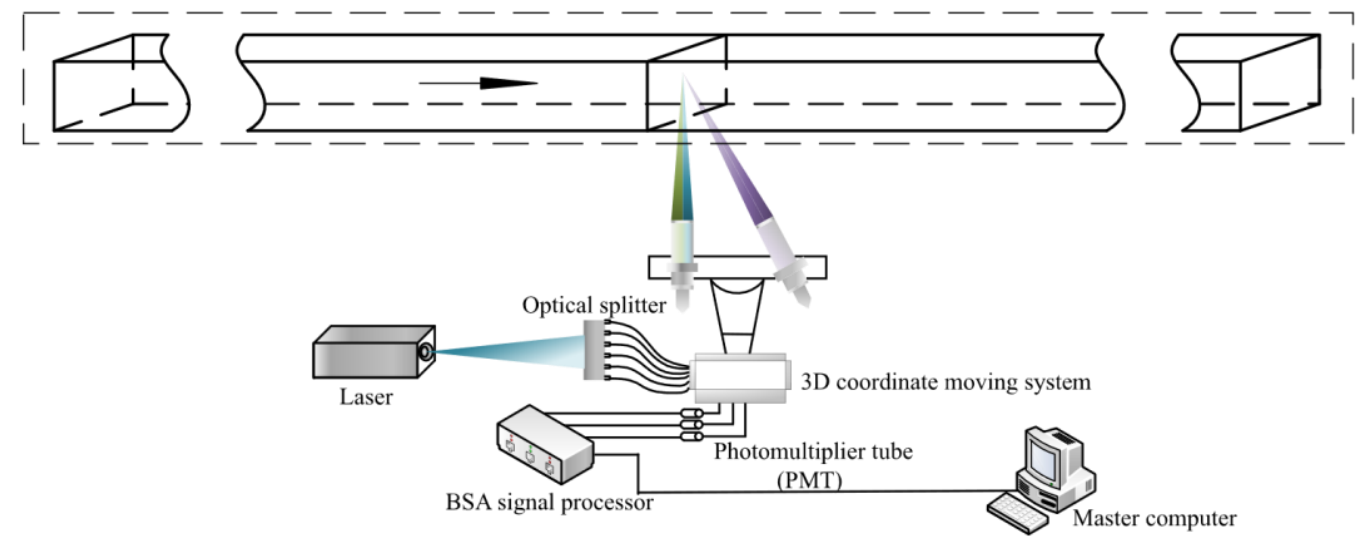

Figure 1. Experimental model and equipment of LDV

\subsection{LDV experimental}

The LDV experiment is a non-contact measurement method with a more accurate test result that reflects the true state of the fluid[18]. The test point is placed at a point where a fully developed flow state is reached[5], and 10 times the cross-section of the air inlet,. The arrangement of the measuring points and the cross-section are shown in Figure 2. The experimental sampling time is 10s. The fan was turned on when all the equipment was ready, and testing started 10 minutes after the fan was on. The experiment was carried out under ideal conditions in which the wind flow was stable, and the environment was free from external interference.

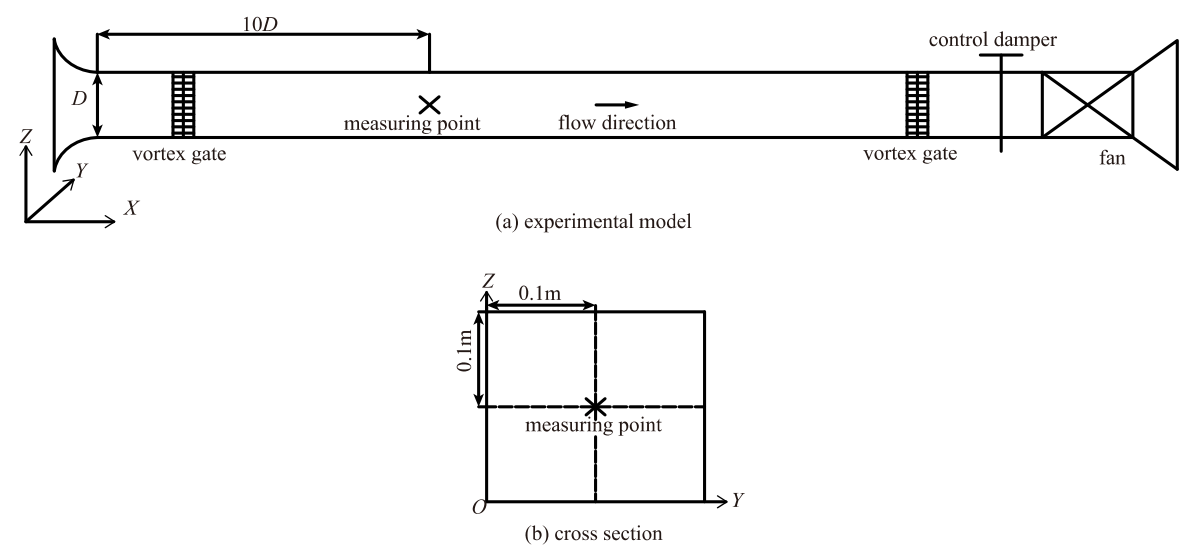

Figure 2. Test model and its cross section

\subsection{Field experiment}

In order to verify the practicality and accuracy of Kalman filter in online monitoring of mine wind speed, field experiments were conducted in the experimental mine of Liaoning Technology University. Figure 3 shows the experimental plan. Experimental mine was a $3 \times 2.5$ rectangular section with a unit of $\mathrm{m}$. The measuring points were placed $60 \mathrm{~m}$ away from the damper 2 and the corner of the roadway, $0.5 \mathrm{~m}$ away from the roof of the roadway and $1.5 \mathrm{~m}$ away from the wall of the tunnel. The wind speed sensor model was used KDF9403, and its test range was $0.4-20 \mathrm{~m} / \mathrm{s}$. The fan ran for more than 10 minutes before collecting data at the measuring point. At this time, the damper 1 was closed, and in order to ensure the stability of the fan as much as possible, the wind window of the 
damper 1 is opened, as shown in Fig. 4(a). The damper 2 was fully opened state, as shown in Fig. 4(b). After the measuring points collected the data for 15 minutes, the damper 1 was fully opened, remaining in the same state as the damper 2, while data collection continued. Data collection continued and the test was terminated after 15 minutes.

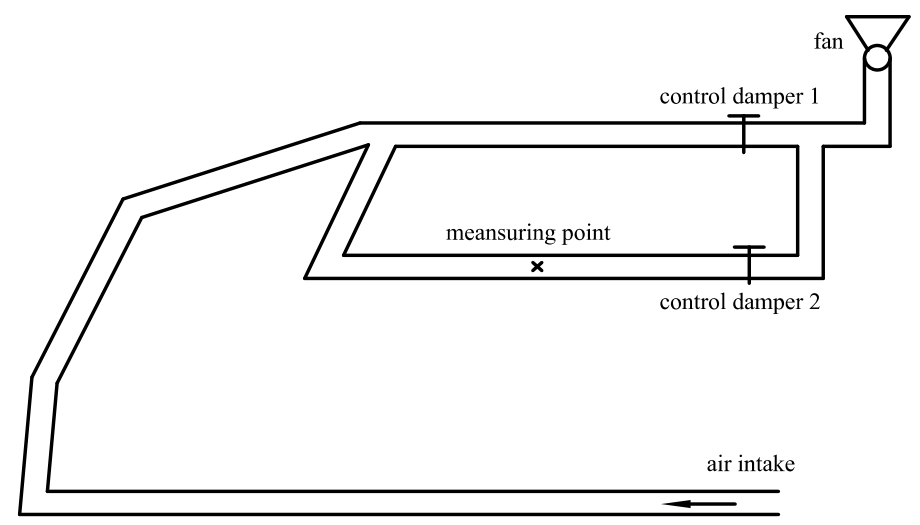

Figure 3. The experiment of mine floor plan

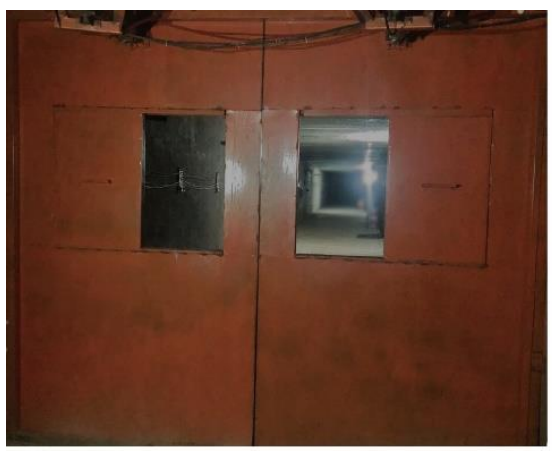

(a)

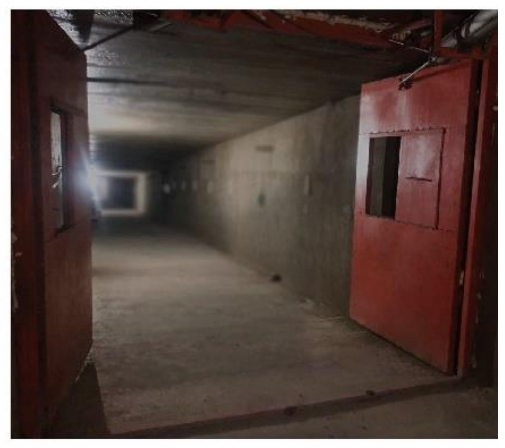

(b)

Figure 4. Field experiment of mine air door

\section{Results}

\subsection{Offline filter process results}

After the Kalman filter offline processing, the average values of wind speed were almost equal to those obtained the LDV test, but the RMSEs were hugely reduced (Table 1). The RMSE was obtained using the mean of wind speed as a reference in the sampling time $\mathrm{T}$. The average value of the wind speed test results in 10 s was $3.308 \mathrm{~m} / \mathrm{s}$, and the RMSE was $0.299 \mathrm{~m} / \mathrm{s}$. There was no outlier in the 1 to $4 \mathrm{~s}$ (Figure 5), RMSE was $0.286 \mathrm{~m} / \mathrm{s}$. There was an outlier in the 4 to $6 \mathrm{~s}$ test (Figure 6), RMSE was $0.381 \mathrm{~m} / \mathrm{s}$. After traditional Kalman filter processing, the average value within $10 \mathrm{~s}$ was $3.308 \mathrm{~m} / \mathrm{s}$ and the RMSE was $0.108 \mathrm{~m} / \mathrm{s}$. the RMSE in $1-4 \mathrm{~s}$ was $0.079 \mathrm{~m} / \mathrm{s}$, while in $4-6 \mathrm{~s}$ was $0.165 \mathrm{~m} / \mathrm{s}$. After adaptive Kalman filter processing, the average value within $10 \mathrm{~s}$ was $3.307 \mathrm{~m} / \mathrm{s}$ and the RMSE was $0.012 \mathrm{~m} / \mathrm{s}$. the RMSE in $1-4 \mathrm{~s}$ was $0.004 \mathrm{~m} / \mathrm{s}$, while in $4-6 \mathrm{~s}$ was $0.018 \mathrm{~m} / \mathrm{s}$.

Table 1. Results of test and Kalman filter signal processing

\begin{tabular}{|c|c|c|c|c|c|c|}
\hline \multirow[b]{2}{*}{ values } & \multicolumn{2}{|c|}{$1-4 \mathrm{~s}$} & \multicolumn{2}{|c|}{$4-6 s$} & \multicolumn{2}{|c|}{$0-10 s$} \\
\hline & $\begin{array}{c}\text { RMSE ( } \\
\mathrm{m} / \mathrm{s})\end{array}$ & mean $(\mathrm{m} / \mathrm{s}$ & $\begin{array}{c}\text { RMSE ( } \\
\mathrm{m} / \mathrm{s})\end{array}$ & $\begin{array}{c}\text { mean }( \\
\mathrm{m} / \mathrm{s})\end{array}$ & $\begin{array}{c}\text { RMSE ( } \\
\mathrm{m} / \mathrm{s})\end{array}$ & $\begin{array}{c}\text { mean }( \\
\mathrm{m} / \mathrm{s})\end{array}$ \\
\hline LDV & 0.286 & 3.307 & 0.381 & 3.297 & 0.299 & 3.308 \\
\hline Traditional filter & 0.076 & 3.310 & 0.165 & 3.299 & 0.108 & 3.308 \\
\hline Adaptive filter & 0.004 & 3.303 & 0.018 & 3.309 & 0.012 & 3.307 \\
\hline
\end{tabular}


Following the traditional Kalman filter process, the wind speed signal tended to be stable, but it was difficult to eliminate the abnormal point in the wind speed signal. After the adaptive Kalman filter processing, the wind speed signal fluctuated around the average value, and the fluctuation range was $3.28 \mathrm{~m} / \mathrm{s}-3.34 \mathrm{~m} / \mathrm{s}$, indicating relatively stable (Figure $5-7$ ).

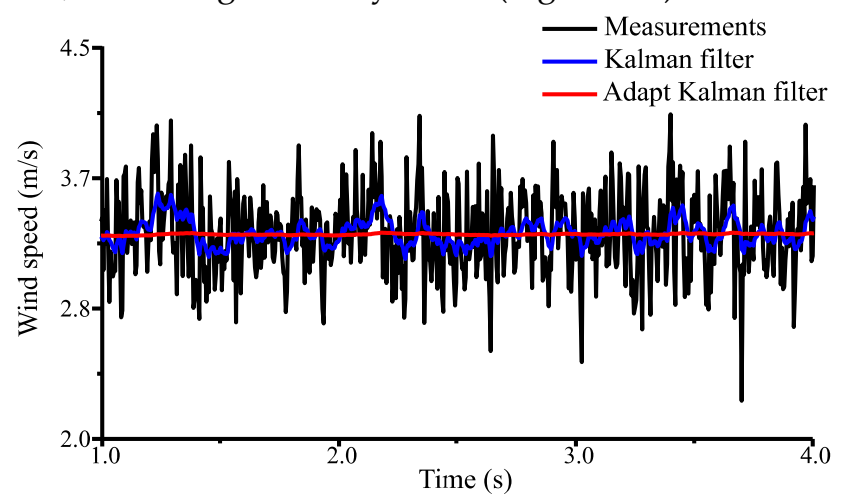

Figure 5. Experiment results without outliers in $1 \mathrm{~s}-4 \mathrm{~s}$ and the consequences of filtering

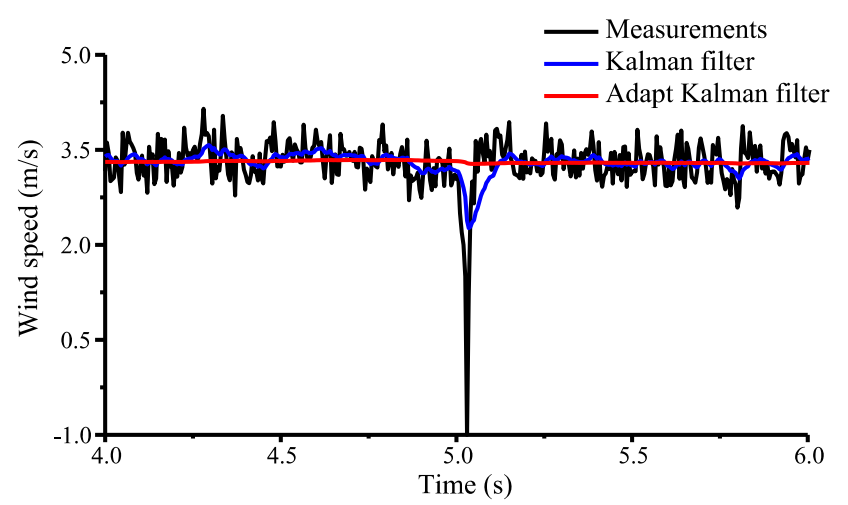

Figure 6. Experiment results with outliers in $4 \mathrm{~s}-6 \mathrm{~s}$ and the consequences of filtering

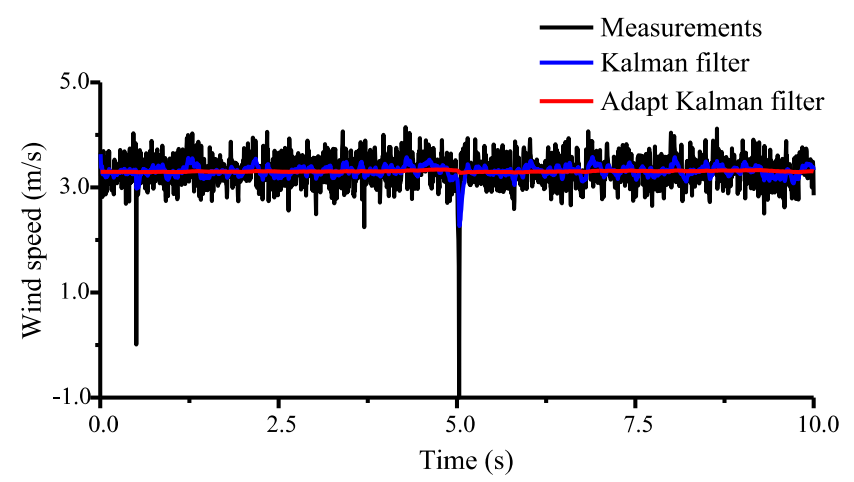

Figure 7. Wind speed signal and the consequences of filtering in $10 \mathrm{~s}$

\subsection{Field experiment results}

The field experiment results showed that even if the system was not adjusted or moved, the signal monitored by the sensor fluctuated largely, and the fluctuation range was $2.4-3.19 \mathrm{~m} / \mathrm{s}$ (Figure $8)$. Before the dampers was closed, the average value of wind speed was $2.83 \mathrm{~m} / \mathrm{s}$, the RMSE was 0.141 $\mathrm{m} / \mathrm{s}$, and the average value of wind speed in the $600-900 \mathrm{~s}$ is $2.83 \mathrm{~m} / \mathrm{s}$, and the RMSE is $0.119 \mathrm{~m} / \mathrm{s}$. Following the adaptive Kalman filter, the mean of wind speed was $2.82 \mathrm{~m} / \mathrm{s}$, and the RMSE was $0.017 \mathrm{~m} / \mathrm{s}$ in the first $5 \mathrm{minutes}$. The average value was $2.83 \mathrm{~m} / \mathrm{s}$, and the RMSE was reduced to $0.009 \mathrm{~m} / \mathrm{s}$ in the 600-900s (Table 2). After the damper 2 was opened, the experiment was continued for 15 minutes. The results showed that the wind speed had a slow downward trend, and the trend of decline after the adaptive Kalman filter treatment was more obvious (Figure 9). After the damper 
was opened for about 2 minute, the wind speed remained at around $0.87 \mathrm{~m} / \mathrm{s}$, and the RMSE decreased by $0.07 \mathrm{~m} / \mathrm{s}$.

Table 2. Real-time monitoring of experimental mine wind speed and results of Kalman filtering

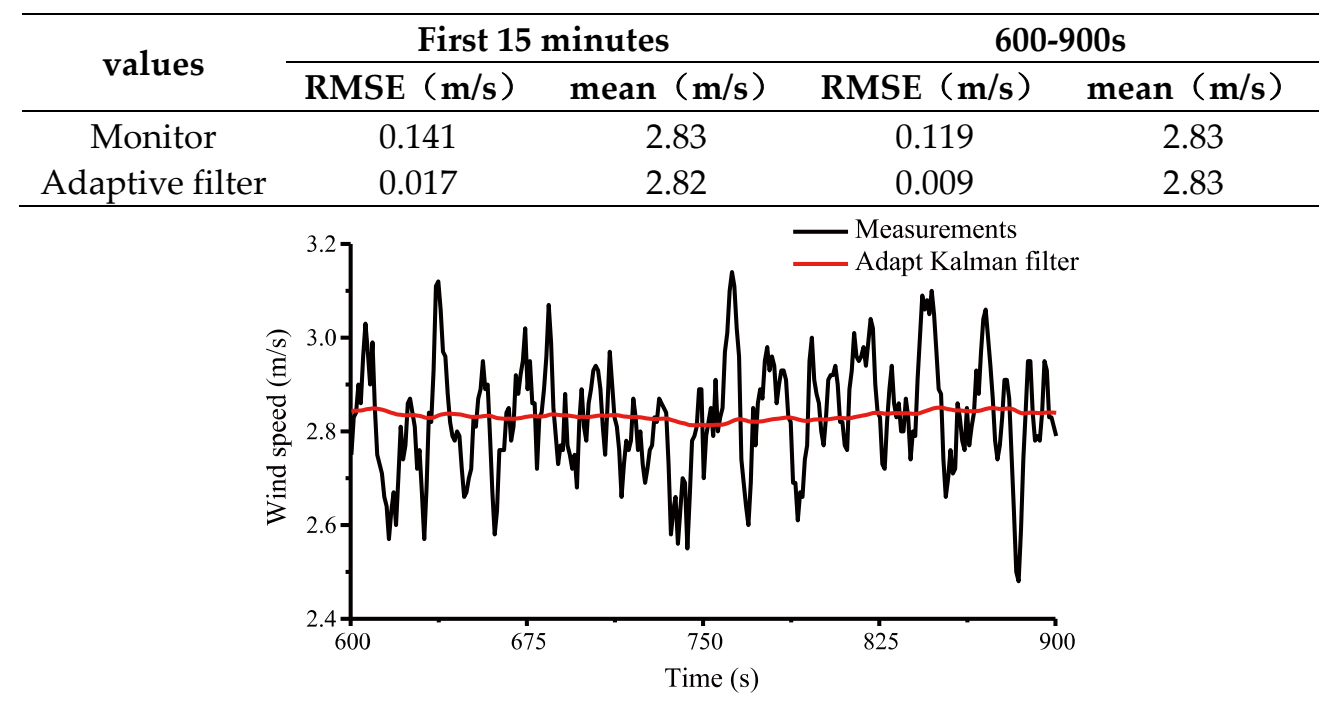

Figure 8. Field monitoring and filtering results in 220s-280s

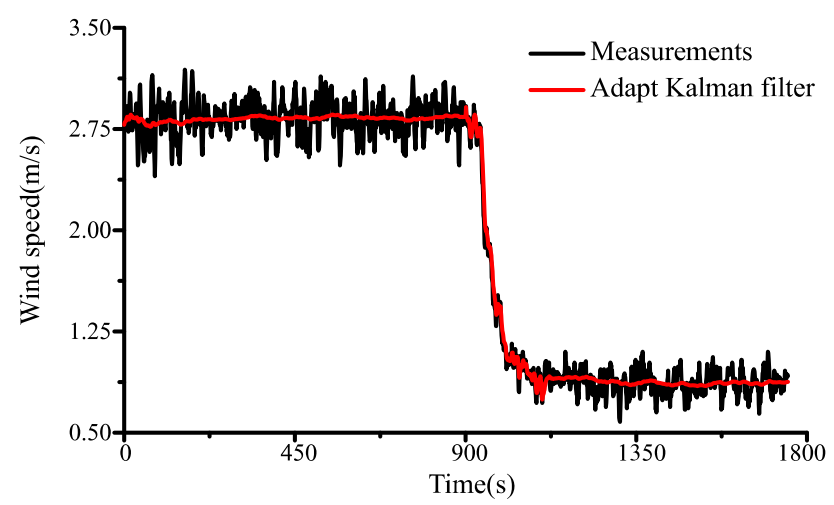

Figure 9. Field monitoring and filtering results in 10 minutes including system changed.

\section{Discussion}

Studies on wind speed test by laser Doppler velocimetry system, the results showed that even in the relatively stable and ideal experimental conditions, the wind speed of the test points in the pipeline also had random fluctuations, and outliers. The reason for the outlier may be that the fan did not run completely stable, or the tracer particles were not observed at the time of data acquisition. The Kalman filter offline processing on the wind speed signal reduced the RMSE, but couldn't completely remove the outlier of the signal. The adaptive Kalman filter could not only reduce the RMSE, but also eliminate the outliers of the wind speed signal. Results showed that the performance of the adaptive Kalman filter is better than the traditional Kalman filter (Figure 6).

The field experiment results showed that there was a random disturbance in the online monitoring wind speed of the measurement points in the tunnel. Even when the system had no changes, the monitored wind speed signals were not so smoothly stable as those obtained under LDV experimental conditions. The reasons for such observations might be voltage instability, which causes changes of the fan operating conditions, or the natural wind flow in the air inlet, which affected the wind speed. The adaptive Kalman filter treatment reduced the RMSE greatly. When the system is changing, the adaptive Kalman filter can quickly reflect the real change process, instead of treating the actual change value as the outlier (Figure 9). Therefore, the adaptive Kalman filter can be applied for mine wind speed online monitoring signal processing. 
Comparison of the RMSEs obtained in the wind speed monitoring and the filter processing revealed that the RMSE at the sampling time without outliers was smaller than that at the sampling time with outliers. When the number of outliers was constant, the longer the sampling time is, the more accurate the experiment results are, which is in line with the law of signal processing [19]. Results proved the reliability of test systems, models, and methods. In the case where the test system does not change, and no outliers occur, the mean value of the monitored speed during the sampling time is generally equal to the mean value of the filtered speed. Data showed that the monitoring methods commonly used in engineering have a certain reliability. However, the occurrence of abnormal points and the time and location of system changes are unknown, therefore, in actual applications, there may be some errors that cause the sensor to alarm under normal conditions, which results in the emergency rescue program, or the alarming system fails to respond to a real emergency condition, which results in safety issues. The wind speed signal filtered by the adaptive Kalman filter, when there is a wild value, in the case that the system does not change, the signal at each monitoring moment is close to the mean of signal at the sampling time. when the system changes, the filtered signal can give a corresponding response. As a result, adaptive Kalman filter can quickly reflect the real wind speed at each measuring point. The wind speed and other parameters at the measuring point can ensure the safety of production at each monitoring time and ensure the safe and effective production of the mine. The Kalman filter predicts the monitored values based on a small number of measurements [9]. Applying the Kalman filter to mine wind speed online monitoring signal processing can provide short-term wind speed online prediction in the event of an accident or failure of the ventilation system, ensure the wind speed monitored at every moment, and it can also provide some reference clues for the rescue work in the accident.

Compared the results of previous researchers' experiments on mine wind speed using LDV, the mine wind speed test conforms to the uncertainty principle. The wind speed signal results conform to the normal distribution, and the signal pulsation has little correlation with the wind speed and sampling time. Their experiment results are consistent with the LDV examination results of this research. The wind speed values in previous study were the average wind speed in the sampling time [5], while the current study used the instantaneous wind speed, which was the wind speed value after Kalman filter treatment at the sampling moment. According to the test results, when the stability of the testing system is free from external interference, the average wind speed obtained by LDV during the sampling time is close to the instantaneous wind speed following Kalman filter treatment. However, when the system is changed, the average wind speed is not equivalent to the instantaneous wind speed. Researchers on vehicle speed monitoring have applied LDV speed measurement system to vehicle speed monitor. The adaptive Kalman filter was used to process the measurement results. Turntable experiments and field test revealed that the adaptive Kalman filter could eliminate the measured outliers, and the RMSE was reduced by $0.370 \mathrm{~cm} / \mathrm{s}$ and $0.021 \mathrm{~m} / \mathrm{s}$, respectively [20]. The difference between the current study and the vehicle speed monitor study is that the measurement of the vehicle running speed was adjusted adaptively for the acceleration variance and the measurement noise variance using the current state space model analysis. Our study was to solve the process noise variance and observation noise variance by using the EM algorithm to find the optimal parameters, thereby achieve the purposes of reducing the errors, eliminating the wild values and the condition of the Kalman filter convergence.

The current study has some shortcomings, parameter optimization of process noise variance and observation noise variance is a non-convex optimization process, and non-convex optimization of parameters using the EM algorithm can easily lead to local optimization. Therefore, the choice of proper initial values for process noise variance and observation noise variance is important in adaptive Kalman filters. Our research analyzed the performance of Kalman filtering. But the reasons for the occurrence of outliers and fluctuations of wind speed were not analyzed thoroughly. This study only investigated treatment of wind speed signals in mine ventilation. To achieve real-time dynamic monitoring and warning of the safety of the ventilation system, it should also monitor other important parameters of the mine ventilation system[21] and perform correlated filtering and noise reduction treatment. 
In the actual production of the mine, circulating ventilation, unreliable ventilation facilities, unsafe ventilation systems, air leakage, insufficient air supply and series ventilation all may cause mine disasters at any time [22]. One of the important parameters for these phenomena is the ventilation air volume of the mine. It is necessary to obtain the accurate wind speed value of the test point. The adaptive Kalman filter can filter the signals monitored online to obtain real-time values by eliminating outliers and reducing RMSE. In addition to the processing of wind speed signals, it is still necessary to study the feasibility of adaptive Kalman filter in processing signals of important ventilation parameters such as gas and wind pressure [23]. Under viable conditions, the scientific theory of mine ventilation safety shall be applied to conduct safety monitoring of mine ventilation system to achieve the purposes of reducing the probability of accidents and ensure the safe and effective production of mines.

\section{Conclusion}

The Kalman filter can eliminate outliers caused by versatile and unpredictable environments and monitoring instrument failures, and it can reduce the random errors of the wind speed signal. As a result, the accuracy of the instantaneous wind speed monitor can be improved. The adaptive Kalman Filter can be reached by adaptive adjustment of process noise variance and observation noise variance. The Kalman filter processing results of the offline signals obtained by the Doppler velocimetry system show that the performance of the adaptive Kalman filter is better than that of the traditional Kalman filter. The online processing of the adaptive Kalman filter for the online wind speed signal monitoring in the experimental mine shows that the adaptive Kalman filter not only improves the accuracy of instantaneous wind speed monitoring but also predicts the short-term wind speed. The adaptive Kalman filter is feasible in the online monitoring signal processing of mine wind speed, which can improve the ability of the ventilation system to identify unsafe factors and states. Therefore, the adaptive Kalman filter is suitable for mine wind speed online monitoring signal processing.

Author Contributions: Conceptualization, Jian Liu; Data curation, De Huang and Lijun Deng; Formal analysis, De Huang; Funding acquisition, Jian Liu; Methodology, Lijun Deng; Resources, Jian Liu; Validation, Xuebing Li and Ying Song; Writing - original draft, De Huang; Writing - review \& editing, Jian Liu, Xuebing Li and Ying Song.

Funding: This research was funded by the National Natural Science Foundation of China (51574142).

Acknowledgments: D.H. is grateful for the experimental equipment of Professor L.J. and the experimental design and support of X.L. and Y.S.

Conflicts of Interest: The authors declare no conflict of interest.

\section{References}

1. Hartman, H. L.; Mutmansky, J. M.; Ramani, R. V.; Wang, Y. J., Mine ventilation and air conditioning. John Wiley \& Sons: 2012.

2. Li, X. B.; Liu, J.; Song, Y.; Wu, G.; Zhang, M. X., On the conversion between the mean airflow velocity and that of the individual point in the underground mine tunnels. Journal of Safety and Environment 2018, 18, (1), 123-128.

3. Song, Y.; Liu, J.; Li, X. B.; Liu, Y. H., Experiment and numerical simulation of average wind speed distribution law of airflow in mine tunnel. China Safety Science Journal 2016, 26, (6), 146-151.

4. $\mathrm{Xu}, \mathrm{J}$. , Fluid machinery and fluid mechanics: 4th International Symposium (4th ISFMFE). Tsinghua University Press and Springer: 2009.

5. Liu, J.; Li, X. B.; Song, Y.; Gao, K.; Deng, L. J., Experimental study on uncertainty mechanism of mine air velocity and pressure with non-external disturbance. Journal of China Coal Society 2016, 41, (06), 14471453.

6. Cheng, D. Q.; Qian, J. S.; Zhou, T.; Li, W. J., A high-speed response linkage model for integrated monitoring system at coal mine based on intellectualized data analysis. Procedia Earth and Planetary Science 2009, 1, (1), 1455-1460. 
7. Huang, Y. R.; Cheng, W. J.; Tang, C. L.; Wang, C. L., Study of multi-agent-based coal mine environmental monitoring system. Ecological Indicators 2015, 51, 79-86.

8. Brown, R. G.; Hwang, P. Y. C., Introduction to random signals and applied Kalman filtering: With MATLAB exercises. 4th ed. / by Robert Grover Brown ed.; John Wiley: 2011.

9. Grewal, M. S.; Andrews, A. P., Kalman filtering: Theory and practice using MATLAB / Mohinder S. Grewal, Angus P. Andrews. 3rd ed. ed.; Wiley: 2008.

10. Candiani, G.; Carnevale, C.; Finzi, G.; Pisoni, E.; Volta, M., A comparison of reanalysis techniques: applying optimal interpolation and Ensemble Kalman Filtering to improve air quality monitoring at mesoscale. The Science of the total environment 2013, 458-460, 7-14.

11. Louka, P.; Galanis, G.; Siebert, N.; Kariniotakis, G.; Katsafados, P.; Pytharoulis, I.; Kallos, G., Improvements in wind speed forecasts for wind power prediction purposes using Kalman filtering. Journal of Wind Engineering and Industrial Aerodynamics 2008, 96, (12), 2348-2362.

12. Şimşek, E. H.; Güleç, F.; Kavuştu, H., Application of Kalman filter to determination of coal liquefaction mechanisms using discrete time models. Fuel 2017, 207, 814-820.

13. Zhang, Q., Adaptive Kalman filter for actuator fault diagnosis. Automatica 2018, 93, 333-342.

14. Kalman, R. E., A New Approach to Linear Filtering and Prediction Problems. Journal of Basic Engineering 1960, 82, (1), 35.

15. Zarchan, P.; Musoff, H., Fundamentals of Kalman filtering: A practical approach / Paul Zarchan, Howard Musoff. 3rd ed. ed.; American Institute of Aeronautics and Astronautics: 2009.

16. Dempster, A. P.; Laird, N. M.; Rubin, D. B., Maximum Likelihood Estimation from incomplete observations. J. Roy. Statist. Soc. B 1977, 39, 1-38.

17. Do, C. B.; Batzoglou, S., What is the expectation maximization algorithm? Nature Biotechnology 2008, 26, (8), 897.

18. Kato, S.; Ito, H.; Ichikawa, T.; Kagami, M., Laser doppler velocimeter. In Google Patents: 1996.

19. Proakis, J. G., Digital signal processing: principles algorithms and applications. Pearson Education India: 2001.

20. Fan, Z.; Sun, Q.; Du, L.; Bai, J.; Liu, J., Application of adaptive Kalman filter in vehicle laser Doppler velocimetry. Optical Fiber Technology 2018, 41, 163-167.

21. Muduli, L.; Mishra, D. P.; Jana, P. K., Application of wireless sensor network for environmental monitoring in underground coal mines: A systematic review. Journal of Network and Computer Applications 2018, $106,48-67$.

22. Ma, J.; Dai, H., A methodology to construct warning index system for coal mine safety based on collaborative management. Safety Science 2017, 93, 86-95.

23. Mishra, P. K.; Pratik; Kumar, M.; Kumar, S.; Mandal, P. K., Wireless real-time sensing platform using vibrating wire-based geotechnical sensor for underground coal mines. Sensors and Actuators A: Physical $2018,269,212-217$. 